\title{
A diffusion Monte Carlo study of small para-Hydrogen clusters
}

\author{
Rafael Guardiola ${ }^{1}$ and Jesús Navarro ${ }^{2}$ \\ 1 Departamento de Fúsica Atómica y Nuclear, Facultad de Física, 46100 Burjassot, Spain \\ ${ }^{2}$ IFIC (CSIC-Universidad de Valencia), Apdo. 22085, 46071 Valencia, Spain
}

(Dated: October 29, 2018)

\begin{abstract}
Ground state energies and chemical potentials of parahydrogen clusters are calculated from 3 to 40 molecules using the diffusion Monte Carlo technique with two different $p-\mathrm{H}_{2}-p-\mathrm{H}_{2}$ interactions. This calculation improves a previous one by the inclusion of three-body correlations in the importance sampling, by the time step adjustement and by a better estimation of the statistical errors. Apart from the cluster with 13 molecules, no other magic clusters are predicted, in contrast with path integral Monte Carlo results.
\end{abstract}

PACS numbers: 67.40.Db, 36.40.-c, 61.46.Bc

Theoretical studies of parahydrogen clusters have attracted a growing interest in the past years, partly motivated by a recent experiment [1] in which Raman scattering was used in cryogenic free jets of the pure gas. Small changes in the frequency near the $Q_{1}(0)$ line of the monomer, were observed and interpreted as intermolecular effects on the intramolecular potential. $\left(p-\mathrm{H}_{2}\right)_{N}$ clusters with $N=2-8$ were clearly identified through frequency shifts ranging from $\Delta \nu=-0.40 \mathrm{~cm}^{-1}$ for $N=2$ to $\Delta \nu=-2.35 \mathrm{~cm}^{-1}$ for $N=8$. The experiment also showed a bump at $N=13, N=33$ and $N=55$, which were interpreted as a signal of magical clusters. However, it is worth mentioning that these three values are actually extrapolations from smaller clusters and presumably are approximate.

Magical numbers appear in classical Lennard-Jones clusters, related to geometrical shapes [2]. Several papers have appeared in the last year with the main objective of checking the magical numbers found in Ref. [1], and/or studying possible superfluidity effects in parahydrogen clusters. Indeed, Path Integral Monte Carlo (PIMC) calculations [3] have found a large superfluid fraction in clusters with $\mathrm{N}=13$ and 18 at temperatures $T \leq 2 \mathrm{~K}$. A superfluid response has been observed in small clusters consisting of a carbonyl sulfide cromophore surrounded by $15-17 p-\mathrm{H}_{2}$ molecules, all within a large helium droplet 44. This has been confirmed by several MC simulations [5, 6, 7, 8, 8] of doped $p$ - $\mathrm{H}_{2}$ clusters.

Systematic studies of $\left(p-\mathrm{H}_{2}\right)_{N}$ clusters, covering the range from $N=3$ to $N=50$ molecules, have been done based on powerful many-body techniques, as difusion Monte Carlo (DMC) [10], PIMC [12, 13, 14], and PIMC adapted to the ground state (PIGS) 11]. Whereas up to $N \simeq 22$ all these calculations are substantially in agreement, for heavier clusters there are noticeable differences between DMC and PIMC results, particularly for $N \geq 26$. PIMC chemical potentials show very prominent peaks at $\mathrm{N}=26,29,34$ and 39 , in contrast with the smooth behavior obtained with DMC.

In this work we present new DMC calculations, improving our previous ones [10] so as to get very precise results within our computational capacity. Specifically, we consider three aspects: the importance sampling func- tion, the time step adjustement and the estimation of the statistical errors.

The DMC procedure is significantly improved when using a good importance sampling wave function, the main effect being the reduction of the variance of the stochastic procedure. We have used a Jastrow function with two- and three-body correlations:

$$
\Phi_{T}=\exp \left(\bar{u}_{2}+u_{3}\right)
$$

where

$$
\bar{u}_{2}=\sum_{i<j}\left[u_{2}\left(r_{i j}\right)+\lambda_{T} \xi^{2}\left(r_{i j}\right) r_{i j}^{2}\right]
$$

with

$$
\begin{aligned}
& u_{2}(r)=-\sum_{i<j}\left[\frac{p_{5}}{r_{i j}^{5}}+\frac{p_{1}}{r_{i j}}\right], \\
& \xi(r)=\exp \left(-\frac{\left(r-s_{T}\right)^{2}}{w_{T}^{2}}\right),
\end{aligned}
$$

and

$$
u_{3}=-\frac{\lambda_{T}}{2} \sum_{\ell} \mathbf{G}_{\ell} \mathbf{G}_{\ell}
$$

with

$$
\mathbf{G}_{\ell}=\sum_{i \neq \ell} \xi\left(r_{l i}\right) \mathbf{r}_{l i}
$$

Indices $i, j, l$ run over the number of molecules in the cluster. This function is described in terms of five variational parameters, $p_{5}, p_{1}, s_{T}, \omega_{T}$, and $\lambda_{T}$. In our previous calculations [10] we used the standard two-body Jastrow function $\Phi_{T}=\exp \left(u_{2}\right)$. The present trial function includes an enlarged the two-body variational space and also three-body correlations in the form suggested in Ref. [19], which still has $\mathcal{O}\left(N^{2}\right)$ computational complexity.

DMC is based in a short-time approximation of the Green's function related to the imaginary time 
Schrödinger equation. In this way, an initial wave function $\Phi_{T}(t=0)$ evolves to the exact ground state wave function $\Psi$ at large $t$ after many short-time steps $\tau$. We have used the $\mathcal{O}\left(\tau^{3}\right)$ approximation to the Green's function as described in Refs. [17, 18], which provides energies $\mathcal{O}\left(\tau^{2}\right)$. The time step adjustment is the following: from calculations at the relative large steps 0.001 and $0.0005 \mathrm{~K}^{-1}$, we obtain the Richardson extrapolated value

$$
\frac{1}{3}(4 E(0.0005)-E(0.001))
$$

based on the $\tau$ expansion $E(\tau)=E(0)+C \tau^{2}+\cdots$. This value turns out to be very close to the calculations with much smaller time steps, as it may be checked in the last three rows of Table [1. This checks that the algorithm behaves as $\mathcal{O}\left(\tau^{2}\right)$, as expected, and suggests to use the value $\tau=0.0001 \mathrm{~K}^{-1}$ for massive calculations with a negligible bias.

TABLE I: Determination of optimal time step $\tau$ from different evaluations of the binding energy $\mathrm{B}(\mathrm{N})$ of several clusters. The row labelled R.E. is the Richardson extrapolated value obtained from the previous two rows. The statistical standard deviation is indicated in parenthesis (error in the final digit shown). Energies and statistical errors are in K.

\begin{tabular}{llll}
\hline \hline$\tau$ & $B(10)$ & $B(20)$ & $B(30)$ \\
0.001 & $183.47(5)$ & $559.28(17)$ & $1006.4(3)$ \\
0.0005 & $185.91(6)$ & $566.56(17)$ & $1020.0(4)$ \\
$R . E$. & $186.72(9)$ & $568.99(28)$ & $1024.5(5)$ \\
0.0001 & $186.93(6)$ & $569.16(12)$ & $1025.2(2)$ \\
0.00002 & $186.72(3)$ & $569.48(7)$ & $1024.8(1)$ \\
\hline \hline
\end{tabular}

A further improvement of the calculation regards the estimate of the statistical error. Because of the sequential Markov chain nature of Monte Carlo algorithms, successive samples are strongly correlated, and the typical way of estimating the variance, $\sigma^{2}=\left\langle H^{2}\right\rangle-\langle H\rangle^{2}$, may be too optimistic. To avoid these correlations we computed a number of times (10, typically), the binding energies, with independent and randomized runs, and estimate the variance from these results. This requires a considerable increasing in computational time, but the obtained standard deviations are very precisely computed. Specifically, we have used 1000 walkers with $10^{5}$ steps plus 20000 stabilization steps in each walker.

Hydrogen molecules interact through weak van der Waals forces that, nevertheless, are sufficiently strong to bound clusters with any number of molecules. Several forms have been derived to describe the $p-\mathrm{H}_{2}-p-\mathrm{H}_{2}$ interaction. Two of them are of particular interest because they combine $a b$ initio properties with properties of the gas (or solid) as well as experimental information from collisions, one due to Silvera and Goldman [15], and the other to Buck et. al. [16], hereafter referred to as SG and BHKOS, respectively. The main difference among them is that the former contains a repulsive long-range term $\left(c_{9} / r^{9}\right)$ with the objective of providing an approxi- mation for the effective potential in a solid. Recent calculations have employed BHKOS potential [10], SG potential [12, 13, 14] or both [11]. We present here results with both interactions.

TABLE II: DMC ground state $\left(\mathrm{p}-\mathrm{H}_{2}\right)_{N}$ binding energies (in $\mathrm{K})$ obtained with BHKOS interaction [16].

\begin{tabular}{rrrrrr}
\hline \hline $\mathrm{N}$ & & $\mathrm{N}$ & & $\mathrm{N}$ & \\
\hline 2 & 4.3114 & 15 & $371.17(3)$ & 28 & $931.46(18)$ \\
3 & $14.66(1)$ & 16 & $408.56(5)$ & 29 & $978.50(10)$ \\
4 & $30.50(1)$ & 17 & $446.68(3)$ & 30 & $1025.66(14)$ \\
5 & $50.33(1)$ & 18 & $486.34(8)$ & 31 & $1074.03(30)$ \\
6 & $73.40(1)$ & 19 & $527.46(8)$ & 32 & $1122.19(23)$ \\
7 & $98.76(1)$ & 20 & $569.72(6)$ & 33 & $1170.42(38)$ \\
8 & $126.16(1)$ & 21 & $612.27(11)$ & 34 & $1219.26(25)$ \\
9 & $155.51(2)$ & 22 & $655.65(12)$ & 35 & $1267.74(35)$ \\
10 & $186.86(3)$ & 23 & $700.50(13)$ & 36 & $1317.87(15)$ \\
11 & $220.98(2)$ & 24 & $745.63(14)$ & 37 & $1366.26(42)$ \\
12 & $257.94(2)$ & 25 & $791.57(16)$ & 38 & $1416.12(22)$ \\
13 & $297.80(9)$ & 26 & $837.88(29)$ & 39 & $1465.55(42)$ \\
14 & $334.24(5)$ & 27 & $884.71(15)$ & 40 & $1516.00(37)$ \\
\hline \hline
\end{tabular}

TABLE III: DMC ground state $\left(\mathrm{p}-\mathrm{H}_{2}\right)_{N}$ binding energies (in $\mathrm{K})$ obtained with the SG interaction [15].

\begin{tabular}{rrrlrr}
\hline \hline $\mathrm{N}$ & & $\mathrm{N}$ & & $\mathrm{N}$ & \\
\hline 2 & 3.8456 & 15 & $341.12(5)$ & 28 & $860.69(12)$ \\
3 & $13.22(1)$ & 16 & $375.81(6)$ & 29 & $904.56(10)$ \\
4 & $27.59(1)$ & 17 & $411.56(4)$ & 30 & $949.06(21)$ \\
5 & $45.73(1)$ & 18 & $448.24(5)$ & 31 & $993.46(12)$ \\
6 & $66.87(1)$ & 19 & $486.31(5)$ & 32 & $1038.04(25)$ \\
7 & $90.14(1)$ & 20 & $525.35(7)$ & 33 & $1083.36(22)$ \\
8 & $115.42(1)$ & 21 & $564.92(10)$ & 34 & $1128.38(32)$ \\
9 & $142.46(2)$ & 22 & $605.40(8)$ & 35 & $1174.29(23)$ \\
10 & $171.41(3)$ & 23 & $646.60(8)$ & 36 & $1220.32(22)$ \\
11 & $202.74(1)$ & 24 & $688.50(11)$ & 37 & $1266.42(20)$ \\
12 & $236.56(4)$ & 25 & $731.02(11)$ & 38 & $1312.22(21)$ \\
13 & $272.53(5)$ & 26 & $774.08(7)$ & 39 & $1358.46(29)$ \\
14 & $306.63(4)$ & 27 & $817.34(14)$ & 40 & $1405.45(27)$ \\
\hline \hline
\end{tabular}

The calculated DMC ground state binding energies are displayed in Tables II and III, for BHKOS and SG potentials, respectively. As usual, the numbers in parenthesis are the errors in the final digit shown, and correspond to the standard deviation. The binding energies for the dimer have been obtained by numerical integration of the Schrödinger equation.

The inclusion of triplet correlations in the importance sampling function leads to a noticeably improvement of the variational energies, as showed in Fig. 1 for BHKOS potential. The DMC energies are basically the same as those of Ref. [10], with slightly more binding in the heavier clusters [20]. The main difference lies in the reduction of the standard deviation by typically a factor of 2 .

The total binding energies grow monotonically with the number of constituents. In order to determine an enhanced stability related to magic sizes it is convenient 
FIG. 1: (Color online) Comparison of the binding energies per molecule obtained with diffusion Monte Carlo (DMC) and a variational Monte Carlo (VMC) calculation based on Eq. (1) with two- and three-body correlations. The interaction is BHKOS. The error bars are within the symbol size.

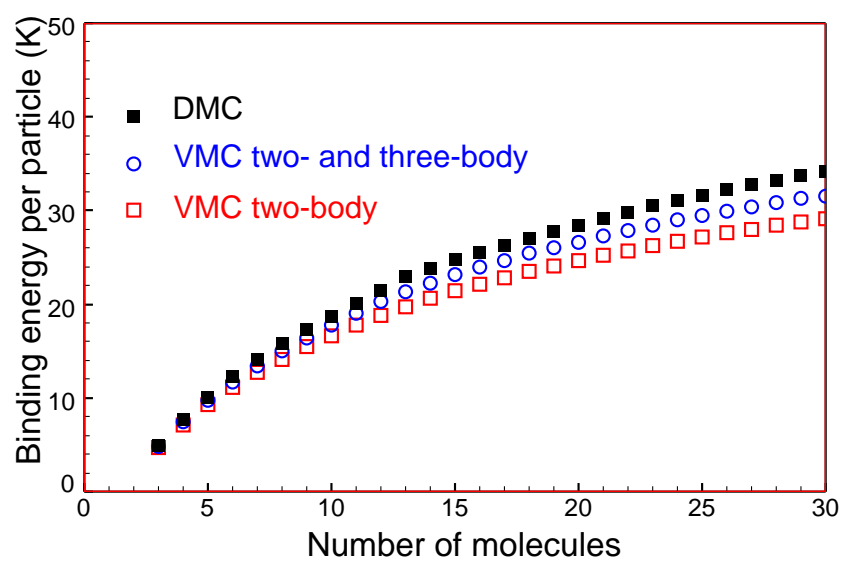

to analyze the variation with $N$ of the dissociation energy or chemical potential, defined from the ground state energies $E(N)$ as

$$
\mu_{N}=E(N-1)-E(N) .
$$

This quantity is plotted in Fig. 2 as a function of the number of molecules $N$. The main physical result of this figure is the presence of a neat peak at $N=13$, indicating the magical character of this cluster. Although the two used interactions give different total energies, the BHKOS potential providing more binding than the SG one, this peak is present for both interactions.

FIG. 2: (Color online) DMC chemical potential (in $\mathrm{K}$ ) of $\left(p-\mathrm{H}_{2}\right)_{N}$ clusters as a function of the number $N$ of molecules. The error bars are within the symbol size.

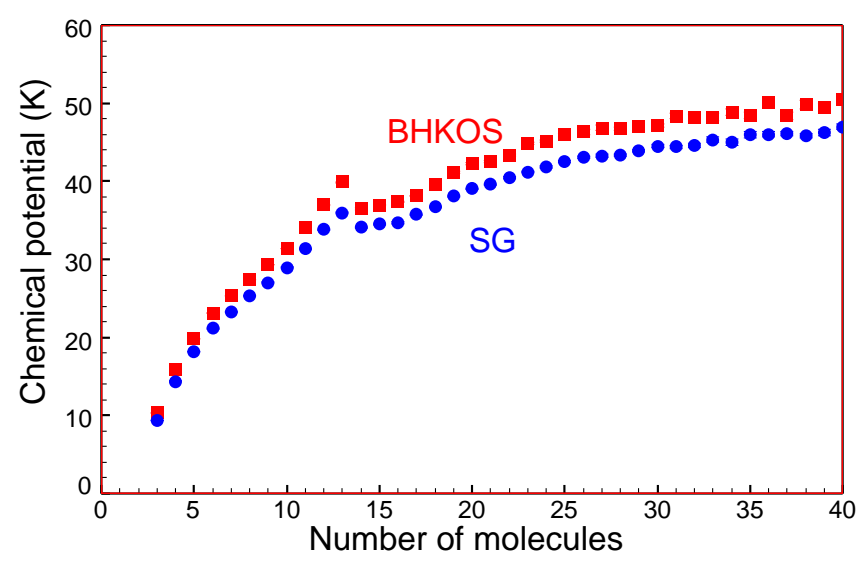

Beyond $N=13$ our calculations do not show any clear signal of local enhancement of the chemical potential. It should be mentioned that even if the relative error of the total energies is around $10^{-4}$, the relative error in the separation energies can be as high as $10^{-2}$, as a consequence of the strong cancelations appearing when computing $\mu$. So, even after our formidable numerical effort, the absolute error of $\mu$ for $N \simeq 40$ may be as high as $0.5 \mathrm{~K}$. Having this fact in mind, the only possible structure, apart from $\mathrm{N}=13$, is $\mathrm{N}=36$ for BHKOS potential. However, $\mu_{36}$ is $\simeq 1.70 \pm 0.6 \mathrm{~K}$ higher than its neighbors $\mu_{35,37}$ and one cannot exclude that it could simply be a statistical fluctuation. Consequently the only magical cluster firmly established here is $\mathrm{N}=13$, independent of the interaction.

FIG. 3: (Color online) DMC and PIMC chemical potentials of $\left(p-\mathrm{H}_{2}\right)_{N}$ clusters as a function of the number $N$ of molecules, calculated with SG interaction [15]. Filled circles and squares are PIMC results of Ref. [14], the solid line corresponds to PIMC results of Ref. [13]. Error bars have not been drawn for the clarity of presentation.

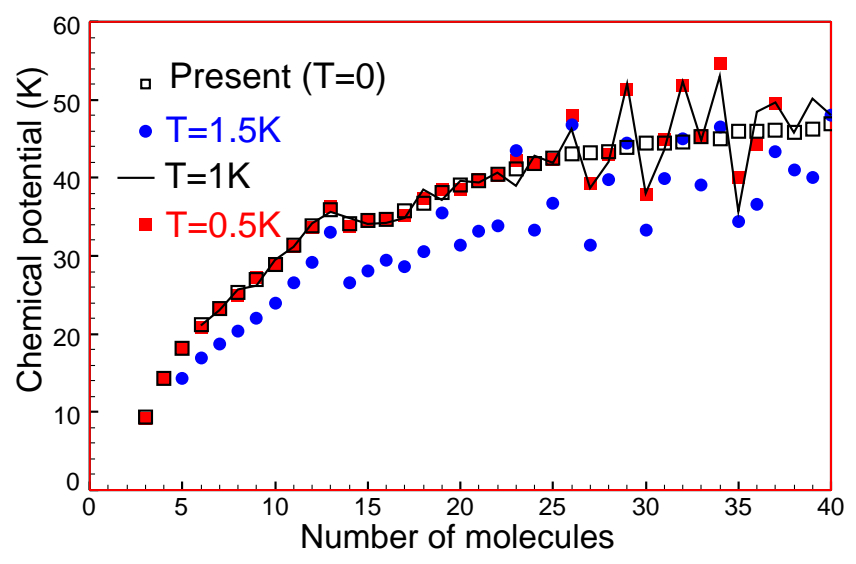

In contrast, PIMC calculations show very prominent peaks at $N=26,29,32,34$ and 39 , as show in Fig. 3. The PIMC results at $T=0.5 \mathrm{~K}$ and $1.5 \mathrm{~K}$ are from Ref. [14], and those at $T=1 \mathrm{~K}$ from Ref. [13]. Up to $N \approx 25$, our DMC results are indistinguishable from the the PIMC ones of Ref. 14 at $T=0.5 \mathrm{~K}$ or those of Ref. 13 at $T=1 \mathrm{~K}$. It is worth noticing that these PIMC results at $T=0.5$ and $1 \mathrm{~K}$ are essentially identical in the calculated range of cluster sizes, with the noticeable exceptions of $N=23,35$ and 36 . However, it should be kept in mind that PIMC error bars have not been drawn in Fig. 3 for the sake of clarity.

The existence of peaks in the chemical potential seems to be related to thermal effects. These could manifest in enhanced stability thresholds at finite temperature, similarly to what has been observed in ${ }^{4} \mathrm{He}$ droplets [21]. But according to Ref. [13] such thermal effects should be associated to a coexistence of solid-like and liquid-like phases, with a dominance of the latter at low $\mathrm{T}$, as a result of both the zero-point motion and quantum permutation exchanges. To this respect it is worth recalling 
that while DMC may be affected by the constraint imposed by the importance sampling function, PIMC has no such constraint. Actually, our importance sampling function is of the type used to describe liquid-like clusters. We have checked that the DMC ground state energy for the magical $\left(p-\mathrm{H}_{2}\right)_{13}$ cluster does not change employing instead an importance sampling function where the molecules are localized at the vertex of the corresponding truncated MacKay icosahedra. This cluster is definitely liquid-like, in agreement with PIMC results. It would be interesting to perform a similar solid-like DMC calculation for $N \geq 26$. Although very computationally demanding, such a calculation could be useful to ascertain the phase of $p-\mathrm{H}_{2}$ clusters in this size region.

The authors acknowledge stimulating conversations with J.P. Toennies and S. Montero and correspondence with J.E. Cuervo. This work is supported by grants FIS2004-00912 (MCyT/FEDER, Spain), and ACOMP07-003 (Generalitat Valenciana, Spain).
[1] G. Tejeda, J.M. Fernández, S. Montero, D. Blume, and J. P. Toennies, Phys. Rev. Lett. 92, 223401 (2004).

[2] F. Baletto and R. Ferrando, Rev. Mod. Phys. 77, 319 (2005).

[3] P. Sindzingre, D.M.Ceperley and M.L.Klein, Phys. Rev. Lett. 67, 1871 (1991).

[4] S. Grebenev, B. Sartakov, J.P. Toennies, and A.F. Vilesov, Science 289, 1532 (2000).

[5] Y. Kwon and K.B. Whaley, Phys. Rev. Lett. 89, 273401 (2002).

[6] F. Paesani, R.E. Zillich and K.B. Whaley, J. Chem. Phys. 119, 11682 (2003).

[7] J. Tang and A.R.W. McKellar, J. Chem. Phys. 121, 3087 (2004).

[8] F. Paesani, R.E. Zillich, Y. Kwon, and K.B. Whaley, J. Chem. Phys. 122, 181106 (2005).

[9] S. Baroni and S. Moroni, ChemPhysChem 6, 1884 (2005].

[10] R. Guardiola and J. Navarro, Phys. Rev. A 74, 025201 (2006).

[11] J.E. Cuervo and P.N. Roy, J. Chem. Phys. 125, 124314 (2006).

[12] F. Mezzacapo and M. Boninsegni, Phys. Rev. Lett. 97,
045301 (2006).

[13] F. Mezzacapo and M. Boninsegni, Phys. Rev. A 75, 033201 (2007).

[14] S.A. Khairallah, M.B. Sevryuk, D.M.Ceperley, and J. P. Toennies, Phys. Rev. Lett. 98, 183401 (2007).

[15] I.F. Silvera, V.V. Goldman, J. Chem. Phys. 69, 4209 (1978)

[16] U. Buck, F. Huisken, A. Kohlhase, D. Otten, and J. Schaeffer, J. Chem. Phys. 78, 4439 (1983)

[17] J. Vrbik and S.M. Rothstein, J. Comput. Phys. 63, 130 (1986).

[18] S.A. Chin, Phys. Rev. A 42, 6991 (1990).

[19] K.E. Schmidt, M.A. Lee, M.H. Kalos, and G.V. Chester, Phys. Rev. Lett. 47, 807 (1981).

[20] There is a misprint in Table I of Ref. [10]. The entry for $B / N$ at $N=23$ should read 30.43(2), instead of the quoted 29.94(2). We acknowledge J.E. Cuervo for having detected it. See also Ref. [13].

[21] R. Brühl, R. Guardiola, A. Kalinin, O. Kornilov, J. Navarro, T. Savas, and J.P. Toennies, Phys. Rev. Lett. 92, 185301 (2004). 